PROCEEDINGS OF THE

AMERICAN MATHEMATICAL SOCIETY

Volume 130, Number 3, Pages 643-651

S 0002-9939(01)06159-7

Article electronically published on September 28, 2001

\title{
A CHARACTERIZATION OF THE HEREDITARY CATEGORIES DERIVED EQUIVALENT TO SOME CATEGORY OF COHERENT SHEAVES ON A WEIGHTED PROJECTIVE LINE
}

\author{
DIETER HAPPEL AND IDUN REITEN \\ (Communicated by Ken Goodearl)
}

\begin{abstract}
Let $\mathcal{H}$ be a connected hereditary abelian category over an algebraically closed field $k$, with finite dimensional homomorphism and extension spaces. There are two main known types of such categories: those derived equivalent to $\bmod \lambda$ for some finite dimensional hereditary $k$-algebra $\lambda$ and those derived equivalent to some category coh $\mathbb{X}$ of coherent sheaves on a weighted projective line $\mathbb{X}$ in the sense of Geigle and Lenzing (1987). The aim of this paper is to give a characterization of the second class in terms of some properties known to hold for these hereditary categories.
\end{abstract}

\section{INTRODUCTION}

Let $k$ be an algebraically closed field and $\mathcal{H}$ a connected abelian $k$-category. We assume that $\mathcal{H}$ is hereditary, that is the Yoneda $\operatorname{Ext}^{2}($,$) vanishes, and we assume$ that $\mathcal{H}$ has finite dimensional homomorphism and extension spaces.

Such categories are of interest in noncommutative algebraic geometry and have been completely classified under the additional assumptions that $\mathcal{H}$ is noetherian and satisfies Serre duality $[\mathrm{RV}]$. They are also of interest in the representation theory of finite dimensional algebras, now under the additional assumption that $\mathcal{H}$ has a tilting object. Recall that an object $T$ in $\mathcal{H}$ is a tilting object if $\operatorname{Ext}^{1}(T, T)=0$ and if $\operatorname{Hom}(T, X)=0=\operatorname{Ext}^{1}(T, X)$ for some object $X$ in $\mathcal{H}$, then $X$ must be 0 . The quasitilted algebras introduced in [HRS] are by definition the endomorphism algebras $\operatorname{End}(T)$, and this class of algebras contains the important classes of tilted and canonical algebras.

Note that a noetherian $\mathcal{H}$ with Serre duality does not usually have a tilting object. Examples are given by categories coh $\mathbb{X}$ of coherent sheaves on a nonsingular projective curve $\mathbb{X}$ different from the projective line $\mathbb{P}^{1}(k)$. On the other hand $\mathcal{H}$ can have a tilting object without being noetherian. An easy example of this is the opposite category of $\operatorname{coh} \mathbb{P}^{1}(k)$.

In this paper we assume that $\mathcal{H}$ has a tilting object. It is then a consequence that $\mathcal{H}$ has almost split sequences, and also Serre duality as studied in [RV] (see [HRS]). When $\mathcal{H}$ is not equivalent to $\bmod \Lambda$ for a finite dimensional hereditary $k$-algebra $\Lambda$, is is known that there is an equivalence $\tau: \mathcal{H} \rightarrow \mathcal{H}$ with the property that if $O \rightarrow A \rightarrow B \rightarrow C \rightarrow O$ is an almost split sequence, then $\tau C \simeq A$ [HRS]. Since

Received by the editors January 13, 2000 and, in revised form, September 12, 2000.

1991 Mathematics Subject Classification. Primary 16B50, 16E10, 16G70, 18E10, 18E30. 
$\mathcal{H}$ has almost split sequences, the familiar concepts of the AR-quiver, and further (standard) stable tubes (which are components of the AR-quiver) from the module theory of finite dimensional algebras carry over to this setting (see [R], [ARS]). For details we refer to section 1 .

There are two main known types of such categories $\mathcal{H}$ with tilting objects; those derived equivalent to $\bmod \Lambda$ for some finite dimensional hereditary $k$-algebra $\Lambda$ and those derived equivalent to some category coh $\mathbb{X}$ of coherent sheaves on a weighted projective line $\mathbb{X}$, in the sense of [GL]. The first class is characterized by the existence of some indecomposable directing object $C$ [HRe1. Recall that $C$ is called directing if it does not lie on a cycle of nonzero nonisomorphisms.

The aim of this paper is to give a characterization of the second class in terms of some properties known to hold for these hereditary categories $[\mathrm{LP}$. The properties are (i) existence of a standard stable tube and (ii) for indecomposable exceptional nondirecting objects $E$ and $F$ in $\mathcal{H}$ we have that $\operatorname{Hom}\left(E, \tau^{-i} F\right)=0$ for $i$ large enough. Recall that $E$ is called exceptional if $\operatorname{Ext}^{1}(E, E)=0$. Note that any summand of a tilting object is exceptional.

Assume that $\mathcal{H}$ is not equivalent to some $\bmod \Lambda$ where $\Lambda$ is a finite dimensional hereditary $k$-algebra. When $\mathcal{H}$ has some simple object, then we know that $\mathcal{H}$ is derived equivalent to some category coh $\mathbb{X}[\mathrm{HRe} 2]$, but the converse does not hold.

We would like to thank the referee for constructive comments.

\section{Standard tubes}

In this section we give some results related to standardness of tubes. In addition to being useful in proving our main theorem, these results may also be of independent interest.

Let $\Lambda$ be a finite dimensional algebra over an algebraically closed field $k$, and let $\underline{r}$ denote the radical of the category $\bmod \Lambda$ of finitely generated $\Lambda$-modules (see $[\mathrm{R}$, ARS]). Recall that a component $\mathcal{C}$ of the AR-quiver is standard if the full subcategory ind $\mathcal{C}$ of $\bmod \Lambda$ whose objects correspond to the vertices of $\mathcal{C}$ is the mesh category of $\mathcal{C}$. The component $\mathcal{C}$ is generalized standard if the restriction of $\underline{r}^{\infty}($,$) to \mathcal{C}$ is zero (see $[\mathrm{R},[\mathrm{ARS}]$ ). The two concepts are known to be equivalent for stable tubes [L1], [S], but there are examples showing that they do not coincide in general [L2].

Now let $\mathcal{C}$ be a component of the AR-quiver of a hereditary abelian $k$-category with tilting object. When $\mathcal{C}$ is a stable tube of rank greater than 1 , which is standard (or generalized standard), then each object $E$ on the border is clearly exceptional. The notions of standard and generalized standard are defined similarly as in the case of finite dimensional algebras.

Assuming only that a stable tube has some exceptional object at the border, rather than assuming that the tube is standard, we get the following useful information on the perpendicular category $E^{\perp}$. The objects of $E^{\perp}$ are by definition the objects $C$ in $\mathcal{H}$ such that $(E, C)=0=\operatorname{Ext}^{1}(E, C)$, where $(E, C)$ denotes $\operatorname{Hom}(E, C)$. We say that $E$ is torsionable if $E$ is the factor of a finite direct sum of copies of some tilting object. Note that this is a condition on the objects in $\mathcal{H}$ without reference to a chosen tilting object $T$. For example all objects in $\bmod \Lambda$ for a hereditary finite dimensional algebra $\Lambda$ are torsionable.

Proposition 1.1. Let $\mathcal{H}$ be a hereditary abelian $k$-category with finite dimensional homomorphism and extension spaces, and having a tilting object. 
Assume that $E$ is an indecomposable torsionable exceptional $\tau$-periodic object of infinite length in $\mathcal{H}$, such that if $O \rightarrow \tau E \rightarrow M \rightarrow E \rightarrow O$ is an almost split sequence, then $M$ is indecomposable. Then we have the following:

(a) $\operatorname{Hom}\left(\tau^{j} E, E\right)=0$ for $0<j<t$, where $t$ is the $\tau$-period of $E$.

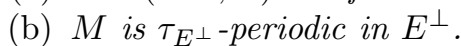

(c) If in addition $E$ is at the border of a stable tube, then the objects on the border are pairwise orthogonal.

Proof. (a) Let $t$ be the $\tau$-period of $E$. Then we have $\tau^{t} E \simeq E$. Consider the almost split sequence $0 \rightarrow \tau E \rightarrow M \rightarrow E \rightarrow 0$. We know from HRe2] that the perpendicular category $E^{\perp}$ is equivalent to $\bmod H$ for some (basic) finite dimensional hereditary $k$-algebra $H$. Further $M$ is in $E^{\perp}$ and $T=H \oplus E$ is a tilting object. The one-point extension algebra $H[M]$ is quasitilted. Since $M$ is indecomposable, it follows that $\operatorname{End}(M) \simeq k$ HRS].

Let $j=1$, and apply the functor $(, M)$ to the above exact sequence to get the exact sequence $0 \rightarrow(E, M) \rightarrow(M, M) \rightarrow(\tau E, M) \rightarrow \operatorname{Ext}^{1}(E, M)$. Since $M \in E^{\perp}$ we have $k \simeq(M, M) \simeq(\tau E, M)$. Now apply $(\tau E$,$) to the almost split sequence, to$ get the exact sequence $0 \rightarrow(\tau E, \tau E) \rightarrow(\tau E, M) \rightarrow(\tau E, E) \rightarrow \operatorname{Ext}^{1}(\tau E, \tau E)=0$. Since $k \simeq(\tau E, \tau E) \simeq(\tau E, M)$, we conclude $(\tau E, E)=0$.

Assume we have proved $\operatorname{Hom}\left(\tau^{j-1} E, E\right)=0$ for some $j>1$. Then since $\operatorname{Ext}^{1}\left(E, \tau^{j} E\right)=0$ we know that any nonzero map $g: \tau^{j} E \rightarrow E$ is a monomorphism or an epimorphism [HRi]. If $g$ is mono, then since $E$ is periodic we get an infinite descending sequence $\cdots \varsubsetneqq E \varsubsetneqq E \varsubsetneqq E$, contradicting the fact that $\operatorname{End}(E)$ is finite dimensional over $k$. If $g$ is an epimorphism, there is an infinite chain of proper epimorphisms $E \stackrel{h}{\rightarrow} E \stackrel{h}{\rightarrow} E \rightarrow \cdots \rightarrow E \rightarrow \cdots$. Then $h$ is not nilpotent, so we have a contradiction. Then we get that $\left(\tau^{j} E, E\right)=0$. This finishes the proof of (a).

(b) Consider the following part of the AR-quiver of $\mathcal{H}$ :

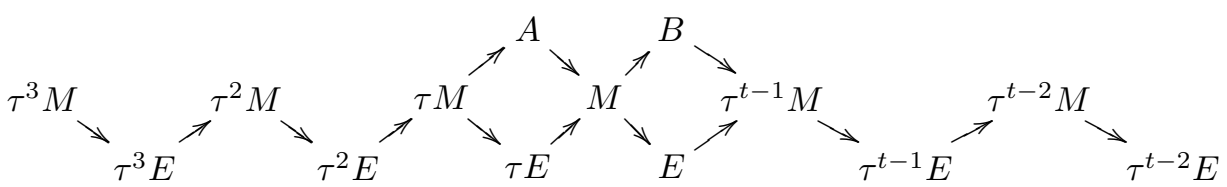

It follows from the above that $\tau^{j} E$ is in $E^{\perp}$ for $j \neq 0,1$. Then $0 \rightarrow \tau^{j+1} E \rightarrow$ $\tau^{j} M \rightarrow \tau^{j} E \rightarrow 0$ is in $E^{\perp}$, and is hence almost split in $E^{\perp}$, for $2 \leq j \leq t-2$. We have the exact sequence $0 \rightarrow \tau^{2} E \rightarrow A \stackrel{v_{1}}{\rightarrow} M \rightarrow 0$ which is in $E^{\perp}$. Consider $f: X \rightarrow M$, where $X$ is indecomposable in $E^{\perp}$, and $f$ is not an isomorphism. We have the almost split sequence $0 \rightarrow \tau M \rightarrow A \oplus \tau E \stackrel{\left(v_{1}, v_{2}\right)}{\longrightarrow} M \rightarrow 0$. Hence there is a map $\left(w_{1}, w_{2}\right): X \rightarrow A \oplus \tau E$ such that $\left(v_{1}, v_{2}\right)\left(w_{1}, w_{2}\right)=f$. Since $(X, \tau E) \simeq D \operatorname{Ext}^{1}(E, X)=0$, where $D$ denotes the duality with respect to the field $k$, we have that $f$ factors through $v_{1}: A \rightarrow M$. Since $\tau^{2} E$ is indecomposable, it follows that the sequence $0 \rightarrow \tau^{2} E \rightarrow A \rightarrow M \rightarrow 0$ is almost split. Consider the exact sequence $0 \rightarrow M \rightarrow X \rightarrow \tau^{t-1} E \rightarrow 0$, which is in $E^{\perp}$, and the almost split sequence $0 \rightarrow M \rightarrow X \oplus E \rightarrow \tau^{t-1} M \rightarrow 0$. Let $f: M \rightarrow C$, with $C$ indecomposable in $E^{\perp}$, be a nonisomorphism. Since $(E, C)=0$ it follows that $g: M \rightarrow B$ is left almost split in $E^{\perp}$, and hence $0 \rightarrow M \rightarrow X \rightarrow \tau^{t-1} E \rightarrow 0$ is almost split in $E^{\perp}$. Combining, we see that $\tau_{E^{\perp}}^{t-1} M \simeq M$, so that $M$ is periodic in $E^{\perp}$.

(c) This follows directly from (a). 


\section{Existence of a Standard tOrsionable tUbe of RANK 1}

Assume as before that $\mathcal{H}$ is a connected hereditary abelian $k$-category with tilting object, and from now on we write "tube" when we mean "stable tube". In this section we assume that $\mathcal{H}$ satisfies the following conditions: (i) There is no simple object in $\mathcal{H}$. (ii) There is some standard tube. (iii) For each indecomposable exceptional torsionable object $E$ the perpendicular category $E^{\perp}$ is equivalent to the module category $\bmod H$ for a (basic) wild finite dimensional hereditary $k$-algebra.

In the derived equivalence class of $\operatorname{coh} \mathbb{X}$, where $\mathbb{X}$ is of tubular type, there are categories $\mathcal{H}$ satisfying (i) and (ii), but not (iii). This is seen by using detailed descriptions in $[\mathrm{H}]$ or $[\mathrm{LS}]$. If $\Lambda$ is the path algebra over $k$ of the quiver

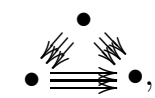

then one can show that there is some $\mathcal{H}$ derived equivalent to $\bmod \Lambda$ which satisfies (i) and (iii), but not (ii).

The aim of this section is to show that under the assumptions (i), (ii) and (iii) any standard tube has rank 1 and is torsionable. We accomplish this through a series of lemmas.

Lemma 2.1. $\mathcal{H}$ is not derived equivalent to any $\bmod \Lambda$ for a finite dimensional hereditary $k$-algebra $\Lambda$, or to any category coh $\mathbb{X}$ of coherent sheaves on a weighted projective line $\mathbb{X}$.

Proof. When $\mathcal{H}$ is derived equivalent to $\bmod \Lambda$ for a finite dimensional hereditary $k$-algebra $\Lambda$, then $\Lambda$ cannot be of wild or of finite type, since in these cases there is no tube. When $\mathcal{H}$ is derived equivalent to $\bmod \Lambda$ for a tame hereditary algebra $\Lambda$, then $\mathcal{H}$ is also derived equivalent to $\operatorname{coh} \mathbb{X}$ for some domestic curve $\mathbb{X}[\mathrm{GL}$. If $\mathcal{H}$ is derived equivalent to some $\operatorname{coh} \mathbb{X}$, then $\operatorname{coh} \mathbb{X}$ is tubular, since otherwise $\mathcal{H}$ has some simple object (see $[\mathrm{H}]$ ). But in this case the module categories $E^{\perp}$ are not wild since they contain tubes.

The following will also be useful.

Lemma 2.2. Let $Y$ be the direct sum of the indecomposable objects at the border of a tube $\mathcal{C}$ and let $E$ be an exceptional indecomposable torsionable object. Then $(E, Y) \neq 0$ or $(Y, E) \neq 0$.

Proof. Assume to the contrary that $(E, Y)=0=(Y, E) \simeq D \operatorname{Ext}^{1}(E, Y)$, where $D$ denotes the duality with respect to the field $k$. Then $Y$ is in $E^{\perp}$, which is equivalent to $\bmod H$ for some wild hereditary $k$-algebra $H$. Since the whole tube $\mathcal{C}$ lies in $E^{\perp}$, and $H$ is wild by assumption (iii), we have a contradiction.

Note that $\mathcal{H}$ is a hereditary abelian $k$-category with tilting object if and only if the opposite category $\mathcal{H}^{o p}$ has the same property.

Lemma 2.3. Let $X$ be at the border of a standard tube $\mathcal{C}$ in $\mathcal{H}$. Then either $X$ is torsionable in $\mathcal{H}$ or $X^{o p}$ is torsionable in $\mathcal{H}^{o p}$.

Proof. Assume that $X$ is not torsionable and let $E$ be an indecomposable exceptional torsionable object. Let $Y$ be the direct sum of the nonisomorphic objects in the $\tau$-orbit of $X$. By possibly replacing $\mathcal{H}$ by $\mathcal{H}^{o p}$ we can assume that $\operatorname{Hom}(E, Y) \neq 0$. By possibly replacing $E$ by an object in the same $\tau$-orbit, we 
can assume that $\operatorname{Hom}(E, X) \neq 0$. We have $E^{\perp}=\bmod H$ for a finite dimensional (basic) hereditary $k$-algebra $H$, and $T=E \oplus H$ is a tilting object HRe2. Consider the corresponding torsion exact sequence $0 \rightarrow t X \rightarrow X \rightarrow X / t X \rightarrow 0$. Then $t X$ is a nonzero proper subobject of $X$.

We claim that $t X$ is exceptional. To see this, note first that $\operatorname{Ext}^{1}(t X, X) \simeq$ $D\left(\tau^{-1} X, t X\right)=0$. For if $\tau^{-1} X \not x X$, then $\left(\tau^{-1} X, X\right)=0$, since $X$ lies in a standard tube, and consequently $\left(\tau^{-1} X, t X\right)=0$. Since the tube $\mathcal{C}$ is standard, we have $\operatorname{End}(X) \simeq k$. If $\tau^{-1} X \simeq X$, then $(X, t X)=0$ since $t X$ is properly contained in $X$. We have the exact sequence

$$
\cdots \rightarrow(t X, X / t X) \rightarrow \operatorname{Ext}^{1}(t X, t X) \rightarrow \operatorname{Ext}^{1}(t X, X) .
$$

Since $(t X, X / t X)=0$ and $\operatorname{Ext}^{1}(t X, X)=0$, it follows that $\operatorname{Ext}^{1}(t X, t X)=0$, so that $t X$ is exceptional.

Since we now know that $X$ has some nonzero torsionable exceptional subobject, we can choose some indecomposable exceptional torsionable subobject $F$ of $X$. Then $F^{\perp}=\bmod H^{\prime}$ for some finite dimensional hereditary $k$-algebra $H^{\prime}$, and $T^{\prime}=$ $F \oplus H^{\prime}$ is a tilting object in $\mathcal{H}$ HRe2].

Let $P$ be indecomposable projective in $F^{\perp}$. Since it follows from Lemma 2.1 that $\mathcal{H}$ is not derived equivalent to $\bmod H_{1}$ for any finite dimensional hereditary $k$-algebra $H_{1}$, we know that the one-point extension $H^{\prime}[\mathrm{M}]$ is quasitilted nontilted, where $M$ is given by the almost split sequence $0 \rightarrow \tau F \rightarrow M \rightarrow F \rightarrow 0$. Hence $M$ is a sincere $H^{\prime}$-module (see $[\mathrm{H}]$ ), so that $\operatorname{Hom}(P, M) \neq 0$.

Now let $f: F^{\prime} \rightarrow X$ be a minimal right add $F$-approximation. Since $X$ is assumed not to be torsionable, $f: F^{\prime} \rightarrow X$ is not surjective. Consider the exact sequences $0 \rightarrow \operatorname{Ker} f \rightarrow F^{\prime} \rightarrow \operatorname{Imf} \rightarrow 0$ and $0 \rightarrow \operatorname{Imf} \rightarrow X \rightarrow$ coker $f \rightarrow 0$. Since $\operatorname{Ext}^{1}\left(F, F^{\prime}\right)=0$ and $\operatorname{Ext}^{1}\left(F, F^{\prime}\right) \rightarrow \operatorname{Ext}^{1}(F, \operatorname{Imf})$ is an epimorphism, it follows that $\operatorname{Ext}^{1}(F, \operatorname{Imf})$ is 0 . Applying the functor $(F$,$) to the second exact sequence gives the$ exact sequence

$$
0 \rightarrow(F, \operatorname{Imf}) \rightarrow(F, X) \rightarrow(F, \text { coker } f) \rightarrow \operatorname{Ext}^{1}(F, X) \rightarrow \operatorname{Ext}^{1}(F, \text { coker } f) \rightarrow 0 .
$$

Since $f^{\prime}: F^{\prime} \rightarrow \operatorname{Imf}$ is a minimal right add $F$-approximation, and $\operatorname{End}(F) \simeq k$, because $F$ is exceptional [HRe2], it follows that $(F, \operatorname{Imf}) \rightarrow(F, X)$ is an isomorphism. Since $\operatorname{Ext}^{1}(F, \operatorname{Imf})=0$, we then have $(F$, coker $f)=0$. It follows that $\operatorname{Ext}^{1}(F, X) \simeq D \operatorname{Hom}\left(\tau^{-1} X, F\right)=0$ since $\operatorname{Hom}\left(\tau^{-1} X, X\right)=0$. Hence we get $\operatorname{Ext}^{1}(F, X)=0$, so that $\operatorname{Ext}^{1}(F, \operatorname{coker} f)=0$. This shows that coker $f$ is in $F^{\perp}$.

Since $X$ is not torsionable, $\tau X$ is also not torsionable, so that $\operatorname{Ext}^{1}\left(F \oplus H^{\prime}, \tau X\right) \neq$ 0 . We have $\operatorname{Ext}^{1}(F, \tau X) \simeq D(X, F)=0$ since $F \subset X$ is a proper inclusion and $\operatorname{End}(F) \cong k$. Hence we have $\operatorname{Ext}^{1}\left(H^{\prime}, \tau X\right) \simeq D\left(X, H^{\prime}\right) \neq 0$, so that there is some indecomposable projective $H^{\prime}$-module $Q$ such that $(X, Q) \neq 0$. Since $\left(F^{\prime}, Q\right)=0$, the exact sequence $F^{\prime} \rightarrow X \rightarrow \operatorname{coker} f \rightarrow 0$ gives that $(\operatorname{coker} f, Q) \neq 0$. Since coker $f$ and $Q$ are in mod $H^{\prime}$, there is some indecomposable projective $H^{\prime}$-module $P^{\prime}$ which is a summand of coker $f$. Since $\operatorname{Ext}^{1}\left(P^{\prime}, F^{\prime}\right)=0$, we have $\operatorname{Ext}^{1}\left(P^{\prime}, \operatorname{Imf}\right)=0$, so that the split inclusion $P^{\prime} \rightarrow$ coker $f$ factors through $X \rightarrow \operatorname{coker} f$. Then $P^{\prime}$ would be a summand of $X$, which is a contradiction.

This shows that $X$ is torsionable.

We can now see that our tube must have rank 1.

Lemma 2.4. Any standard tube has rank 1. 
Proof. Assume that there is some standard tube of rank greater than 1 , and let $F$ be at the border of this tube. Since the tube is standard, $F$ is exceptional, and it follows from Lemma 2.3 that we can assume that $F$ is torsionable. Since we have assumed that $\mathcal{H}$ has no simple object, $F^{\perp}=\bmod H$ would contain a periodic $H$ module by Proposition 1.1 which is a contradiction to $H$ being wild. This finishes the proof.

\section{The MAIN RESUlT}

The aim of this section is to prove our desired characterization of the hereditary $k$-categories derived equivalent to some category $\operatorname{coh} \mathbb{X}$.

We first give two preliminary results. For these we assume in addition to our assumptions on hereditary $k$-categories in section 2 that we have some torsionable standard tube of rank one, not just some standard tube.

Lemma 3.1. Under the above assumptions, let $X$ be an indecomposable object at the border of some torsionable standard tube of rank 1 .

Then there is some indecomposable exceptional object $E$ with $\operatorname{Hom}(E, X) \neq 0$ and $X$ not in Fac $E$, where the objects of Fac $E$ are the factors of finite direct sums of copies of $E$.

Proof. Assume to the contrary that whenever $\operatorname{Hom}(E, X) \neq 0$ for some indecomposable exceptional object $E$, then $X$ is in Fac $E$. Since $\operatorname{End}(E) \simeq k$ [HRe2, this implies $\operatorname{Hom}(X, E)=0$, and hence $l(E)=\operatorname{dim}_{k} \operatorname{Hom}(E, X)-\operatorname{dim}_{k} \operatorname{Hom}(X, E)>0$. We choose $E$ such that $l(E)=a>0$ is smallest possible.

Let $f: E^{t} \rightarrow X$ be a minimal right add $E$-approximation, which is necessarily surjective. The perpendicular category $E^{\perp}$ is equivalent to $\bmod H$ for some finite dimensional (basic) hereditary $k$-algebra $H$, and $T=E \oplus H$ is a tilting object [HRe2]. Consider the exact sequence $0 \rightarrow K \rightarrow E^{t} \stackrel{f}{\rightarrow} X \rightarrow 0$. Applying the functor $(E$, to this exact sequence gives rise to the exact sequence $0 \rightarrow(E, K) \rightarrow\left(E, E^{t}\right) \rightarrow$ $(E, X) \rightarrow \operatorname{Ext}^{1}(E, K) \rightarrow \operatorname{Ext}^{1}\left(E, E^{t}\right) \rightarrow \operatorname{Ext}^{1}(E, X) \rightarrow 0$. Since $\operatorname{End}(E) \simeq k$, it follows that $K$ is in $E^{\perp}$. Hence the exact sequence $0 \rightarrow K \rightarrow E^{t} \stackrel{f}{\rightarrow} X \rightarrow 0$ is in $\operatorname{Fac} T$, so that $0 \rightarrow(T, K) \rightarrow\left(T, E^{t}\right) \rightarrow(T, X) \rightarrow 0$ is exact. Now $(T, E)=$ $(k, M$, id $)$ as a $\left(\begin{array}{cc}k & 0 \\ M & H\end{array}\right)$-module, and $(T, X)=(\operatorname{Hom}(E, X), \operatorname{Hom}(H, X), f)$, where $\operatorname{Hom}(E, X)=k^{t}$ for some $t, \operatorname{Hom}(H, X)=I$ is in $\bmod H$ and $f: M^{t} \rightarrow I$ is an $H$ homomorphism. The map $f: M^{t} \rightarrow I$ is surjective since id: $M \rightarrow M$ is surjective. The tilting object $T$ gives rise to a torsion pair $(\mathcal{T}, \mathcal{F})$ in $\mathcal{H}$, where $\mathcal{T}=\operatorname{Fac} T$. We have a split torsion pair $\left(\mathcal{R}^{\prime}, \mathcal{L}^{\prime}\right)$ in $\bmod H[M]$, and $(T):, \mathcal{T} \rightarrow \mathcal{L}^{\prime}$ is an equivalence of categories (see [HRS]). Then the almost split sequence $0 \rightarrow X \rightarrow Y \rightarrow X \rightarrow 0$ in $\mathcal{H}$ lies in $\mathcal{T}$, and hence $0 \rightarrow(T, X) \rightarrow(T, Y) \rightarrow(T, X) \rightarrow 0$ is an exact sequence in $\bmod H[M]$, which lies in $\mathcal{L}^{\prime}$. It is then almost split, and hence $D \operatorname{Tr}(T, X) \simeq(T, X)$, where $\operatorname{Tr}$ denotes the transpose.

We now show that the $H$-module $I$ is injective. Consider the minimal projective $H[M]$-resolution $0 \rightarrow(0, K, 0) \rightarrow\left(k^{t}, M^{t}\right.$, id $) \rightarrow\left(k^{t}, I, f\right) \rightarrow 0$. Using that $D \operatorname{Tr}(T, X) \simeq(T, X)$, we get a minimal injective resolution $0 \rightarrow\left(k^{t}, I, f\right) \rightarrow$ $\left(k^{r}, I(K / \underline{r} K), g\right) \rightarrow\left(k^{t}, 0,0\right) \rightarrow 0$, where $I(K / \underline{r} K)$ denotes the injective envelope of $K / \underline{r} K$. Hence $I$ is isomorphic to $I(K / \underline{r} K)$, and is hence an injective $H$-module.

Since $f: M^{t} \rightarrow I$ is surjective, we see that $\operatorname{Hom}_{H[M]}((T, X), Z)=0$ for all $Z$ in $\bmod H$. Note that $\bmod H$ is a subcategory of both $\mathcal{H}$ and $\bmod H[M]$, and with such an identification $(T$,$) acts as the identity on \bmod H \subset \mathcal{H}$. Hence we also have 
$\operatorname{Hom}_{\mathcal{H}}(X, Z)=0$ for $Z$ in $\bmod H$. This shows that $l(Z)=\operatorname{dim}_{k} \operatorname{Hom}(Z, X)-$ $\operatorname{dim}_{k} \operatorname{Hom}(X, Z) \geq 0$. Let $Z$ be in $\bmod H$, and let $S$ be a simple submodule of $Z$. Then $S$ is exceptional and also torsionable since it is in Fac $H \subset$ Fac $T$. Hence we have $l(S)>0$ by Lemma [2.2, and consequently $l(Z)>0$. In particular $l(S) \geq a$ for each simple submodule $S$ of $M$. Since $l(E)=a=l(\tau E)$, we have $l(M)=2 a$, and hence $\operatorname{dim}_{k} \operatorname{soc} M \leq 2$, where $\operatorname{soc} M$ denotes the socle of $M$. Since by assumption $\mathcal{H}$ is not derived equivalent to any $\bmod \Lambda$ for a finite dimensional hereditary $k$ algebra $\Lambda$, then $H[M]$ is not tilted. Hence we have $\operatorname{dim}_{k}(\operatorname{soc} M)=2$, since $M$ is indecomposable $[\mathrm{H}$. But then we have $l(M / \operatorname{soc} M)=0$, which is a contradiction. This finishes the proof of the lemma.

Lemma 3.2. Under the above assumptions on the hereditary $k$-category $\mathcal{H}$, it follows that $\mathcal{H}$ does not satisfy condition ( $*$ ): If $E$ and $F$ are indecomposable exceptional nondirecting objects, there is some integer $n$ such that $\operatorname{Hom}\left(E, \tau^{-i} F\right)=0$ for $i>n$.

Proof. Let $X$ be at the border of a torsionable standard tube of rank 1. By Lemma 3.1 we can choose some indecomposable exceptional object $E$ such that $\operatorname{Hom}(E, X) \neq 0$ and $X$ is not in $\operatorname{Fac} E$.

Consider the exact sequence $0 \rightarrow \operatorname{Ker} f \rightarrow E^{t} \stackrel{f}{\rightarrow} X \rightarrow$ coker $f \rightarrow 0$, where $f: E^{t} \rightarrow X$ is a minimal right add $E$-approximation. We know that $f \neq 0$ and coker $f \neq 0$. We have the associated exact sequences $0 \rightarrow \operatorname{Imf} \rightarrow X \rightarrow$ coker $f \rightarrow 0$ and $0 \rightarrow \operatorname{Ker} f \rightarrow E^{t} \rightarrow \operatorname{Imf} \rightarrow 0$.

We first want to show that coker $f$ is an exceptional object in $E^{\perp}$. As before we have $(E$, coker $f)=0=\operatorname{Ext}^{1}(E$, coker $f)$, so that coker $f$ is in $E^{\perp}$. We have the exact sequence $(X, \operatorname{coker} f) \rightarrow(\operatorname{Imf}, \operatorname{coker} f) \rightarrow \operatorname{Ext}^{1}(\operatorname{coker} f$, coker $f) \rightarrow$ $\operatorname{Ext}^{1}(X, \operatorname{coker} f) \rightarrow \operatorname{Ext}^{1}(\operatorname{Imf}, \operatorname{coker} f) \rightarrow 0$. Since $\left(E^{t}, \operatorname{coker} f\right)=0$, we have $(\operatorname{Imf}$, coker $f)=0$. Since $\tau X \simeq X$, we have $\operatorname{Ext}^{1}(X$, coker $f) \simeq D(\operatorname{coker} f, X)$. If (coker $f, X) \neq 0$, then the composition $X \rightarrow \operatorname{coker} f \rightarrow X$ is a nonzero map which is not an isomorphism. But $\operatorname{End}(X) \simeq k$ since $X$ lies at the border of a standard tube, and hence we have a contradiction. This shows that $\operatorname{Ext}^{1}(X, \operatorname{coker} f)=0$, and hence $\operatorname{Ext}^{1}(\operatorname{coker} f, \operatorname{coker} f)=0$.

Let $C$ be an indecomposable summand of coker $f$. Then $C$ is exceptional by what we just proved, and $C$ is torsionable since $X$ is torsionable. Assume first that $C$ lies in a tube. We then claim that there is an indecomposable exceptional torsionable object at the border of this tube: Clearly all $\tau^{i} C$ are also exceptional and torsionable. If $C$ is not at the border, we have an almost split sequence $0 \rightarrow$ $\tau C \rightarrow A \oplus B \rightarrow C \rightarrow 0$, where $A$ and $B$ are indecomposable and the induced map $A \rightarrow C$ is a monomorphism. If $\operatorname{Ext}^{1}(A, A) \neq 0$, then $\operatorname{Hom}\left(\tau^{-1} A, A\right) \neq 0$. Hence the composition $C \rightarrow \tau^{-1} A \rightarrow A \rightarrow C$ gives a nonzero map which is not an isomorphism, since $C \rightarrow \tau^{-1} A$ is an epimorphism. Continuing this way we obtain that all objects at the border are exceptional. We replace $C$ by an object at the border which is a factor of $C$. Hence we still have that the new object is torsionable. Since $C$ automatically has infinite length, $C^{\perp}$ is equivalent to $\bmod H$ for some hereditary finite dimensional wild $k$-algebra $H$. If $0 \rightarrow \tau C \rightarrow F \rightarrow C \rightarrow 0$ is almost split, it follows from Proposition 1.1 that $F$ is a periodic $H$-module, which gives a contradiction.

We can now assume that $C$ has infinite $\tau$-period. Since $\tau X \simeq X$, we have an epimorphism $X \rightarrow \tau^{i} C$ for each $i$, and hence a nonzero map $E \rightarrow \tau^{i} C$ for each 
$i$. The modules $E$ and $C$ are both indecomposable exceptional. Since by Lemma $2.1 \mathcal{H}$ is not derived equivalent to $\bmod \Lambda$ for some finite dimensional hereditary $k$-algebra $\Lambda$, we know that $E$ and $C$ are not directing [HRe1. This shows that condition $(*)$ does not hold, and the proof is finished.

We are now in the position to prove our main result.

Theorem 3.3. The following are equivalent for a hereditary abelian $k$-category $\mathcal{H}$ with finite dimensional homomorphism and extension spaces, and having a tilting object:

(a) $\mathcal{H}$ is derived equivalent to some coh $\mathbb{X}$.

(b) (i) There exists a standard stable tube.

(ii) For indecomposable exceptional nondirecting objects $E$ and $F$ with infinite $\tau$-orbit there is some integer $n$ such that $\operatorname{Hom}\left(E, \tau^{-i} F\right)=0$ for $i>n$.

Proof. (a) $\Rightarrow$ (b). Properties (i) and (ii) are known to hold for the hereditary categories derived equivalent to some coh $\mathbb{X}[\mathrm{LP}$.

(b) $\Rightarrow$ (a). Assume that (i) and (ii) are satisfied. If $\mathcal{H}$ is derived equivalent to some $\bmod \Lambda$ for $\Lambda$ a finite dimensional hereditary $k$-algebra $\Lambda$, then $\Lambda$ must be tame since $\mathcal{H}$ has a tube (see $[\mathrm{R}]$ ). Hence $\mathcal{H}$ is derived equivalent to some $\operatorname{coh} \mathbb{X}$. If $\mathcal{H}$ has a simple object, we are then done by [HRe2].

Assume now that $\mathcal{H}$ has no simple object. Let $E$ be an indecomposable exceptional torsionable object. Then $E^{\perp}=\bmod H$ for a finite dimensional hereditary $k$-algebra $H$. If $H$ is of finite type, we know that the quasitilted algebra $H[M]$ is tilted, where $M$ is the middle term of the almost split sequence with right hand term $E$ [HRS]. Then $\mathcal{H}$ is equivalent to $\bmod \Lambda$ for a finite dimensional hereditary $k$-algebra $\Lambda$. Since $\mathcal{H}$ has a tube by assumption, $\Lambda$ must be tame, and hence $\mathcal{H}$ is derived equivalent to $\operatorname{coh} \mathbb{X}$ for some domestic curve $\mathbb{X}[\mathrm{GL}$. If $H$ is tame, then since $H[M]$ is quasitilted, $M$ is either preprojective, preinjective or simple regular. In the first two cases, $H[M]$ is tilted [HRS]. Using the same argument as above, it follows that $\mathcal{H}$ is derived equivalent to $\operatorname{coh} \mathbb{X}$ for some domestic curve $\mathbb{X}$. If $M$ is simple regular, then $H[M]$ is either tilted from a tame hereditary algebra $[R]$ or $H[M]$ can be obtained as the endomorphism ring of a tilting object for some $\operatorname{coh} \mathbb{X}[\mathrm{LM}]$. In both cases we have that $\mathcal{H}$ is derived equivalent to some $\operatorname{coh} \mathbb{X}$. If $H$ is wild, the standard assumptions from section 2 are satisfied. By the results of section 2 also the standard assumptions from section 3 are valid. Then we get a contradiction to (ii), and we are done.

\section{REFERENCES}

[ARS] M. Auslander, I. Reiten and S.O. Smalø, Representation theory of Artin algebras, Cambridge Univ. Press, 1995. MR 96c:16015

[GL] W. Geigle and H. Lenzing, A class of weighted projective curves arising in representation theory of finite dimensional algebras, in: Singularities, representations of algebras and vector bundles, Springer Lecture Notes 1273 (1987) 265-297. MR 89b:14049

$[\mathrm{H}]$ D. Happel, Quasitilted algebras, Proc. ICRA VIII (Trondheim), CMS Conf. Proc., Vol. 23, Algebras and modules I (1998) 55-83. MR 99k:16023

[HRe1] D. Happel and I. Reiten, Directing objects in hereditary categories, Proc. Seattle Conf. on representation theory, Contemp. Math., Vol. 229 (1998) 169-179. MR 2000a:16018

[HRe2] D. Happel and I. Reiten, Hereditary categories with tilting object, Math. Z. 232, 559-588 (1999). MR 2001d:18012

[HRS] D. Happel, I. Reiten and S. O. Smalø, Tilting in abelian categories and quasitilted algebras, Memoirs Amer. Math. Soc. 575 (1996). MR 97j:16009 
[HRi] D. Happel and C. M. Ringel, Tilted algebras, Trans. Amer. Math. Soc. 274 (1982) 399-443. MR 84d:16027

[LM] H. Lenzing and H. Meltzer, Tilting sheaves and concealed canonical algebras, "Representations of algebras", CMS Conf. Proc. vol. 18, CMS/AMS (1996) 455-473. MR 97f:16026

[LP] H. Lenzing and J. A. de la Peña, Wild canonical algebras, Math. Z., 224, no.3, 403-425 (1997). MR 99a:16012

[L1] S. Liu, Infinite radicals in standard Auslander-Reiten components, J. Algebra 166 (1994) 245-254. MR 95g:16014

[L2] S. Liu, Shapes of connected components of Auslander-Reiten quivers of Artin algebras, CMS Conf. Proc. 16 (ICRA VII, Mexico)(1996) 109-137. MR 97e:16037

[LS] H. Lenzing and A. Skowronski, Quasitilted algebras of canonical type, Colloq. Math. 71 (1996), no.161-181. MR 97j:16019

[R] C. M. Ringel, Tame algebras and quadratic forms, Springer Lecture Notes in Math. 1099, Heidelberg 1984. MR 87f:16027

[RV] I. Reiten and M. Van den Bergh, Noetherian hereditary categories with Serre duality.

[S] A. Skowronski, On semiregular Auslander-Reiten components, Associative rings and algebras.

Fakultät für Mathematik, Technische Universität Chemnitz, D-09107 Chemnitz, GerMANY

E-mail address: happel@mathematik.tu-chemnitz.de

Department of Mathematical Sciences, Norwegian University of Science and TechNOLOGY, 7491 TRONDHEIM, NORWAY

E-mail address: idunr@math.ntnu.no 